\title{
Effect of Repeated Wetting-Drying-Freezing-Thawing Cycles on the Mechanic Properties and Pore Characteristics of Compacted Loess
}

\author{
Fei Wang $\mathbb{D}^{1},{ }^{1}$ Guoyu Li $\mathbb{D}^{2}$, Wei Ma $\mathbb{D}^{2},{ }^{2}$ Yanhu Mu $\mathbb{D}^{2},{ }^{2}$ Zhiwei Zhou, ${ }^{2}$ Jun Zhang, \\ Dun Chen, ${ }^{2}$ and Jinshuai Zhao ${ }^{1}$ \\ ${ }^{1}$ Faculty of Civil Engineering and Mechanics, Jiangsu University, Zhenjiang 212013, China \\ ${ }^{2}$ State Key Laboratory of Frozen Soil Engineering, Northwest Institute of Eco-Environment and Resources, \\ Chinese Academy of Sciences, Lanzhou 730000, China
}

Correspondence should be addressed to Guoyu Li; guoyuli@lzb.ac.cn

Received 11 September 2020; Revised 12 November 2020; Accepted 8 December 2020; Published 19 December 2020

Academic Editor: Chunshun Zhang

Copyright (c) 2020 Fei Wang et al. This is an open access article distributed under the Creative Commons Attribution License, which permits unrestricted use, distribution, and reproduction in any medium, provided the original work is properly cited.

A series of laboratory experiments, including oedometer tests, direct shear tests, and mercury intrusion porosity (MIP) tests, were conducted to investigate the effects of repeated wetting-drying-freezing-thawing (WDFT) cycles on the mechanic behaviors and pore characteristics of compacted loess. The results of mechanical tests indicate that the WDFT weathering can cause significant deterioration of mechanical properties for compacted loess. As the number of treatment cycles increases, vertical compression strain and coefficient of collapsibility of the loess specimens increase while the cohesion decreases. The compacted and noncollapsible loess specimen exhibits collapse again after the 7 WDFT cycles. The results of MIP tests show that WDFT cycles have a main influence on the pores with a pore diameter of $1 \sim 35 \mu \mathrm{m}$ between the soil aggregates, and medium pore contents (10 35 $\mu \mathrm{m})$ increase significantly with the increasing number of WDFT cycles. By comparing compression and collapse characteristics of the loess specimens subjected to wetting-drying, freeze-thaw, and WDFT cycles, we found that the dry-wet action plays the dominant role in the deterioration of engineering properties of compacted loess during WDFT cycles.

\section{Introduction}

Loess soil is a type of quaternary aeolian deposits composed predominantly of particles in the range of $0.005 \sim 0.05 \mathrm{~mm}$. It has characteristics of high porosity, low density, high carbonate content, and open and metastable structure. Due to these characteristics, the loess can exhibit a sudden decrease in total volume upon wetting and overburden pressure which causes various problems to man-made infrastructures built on loess soils [1].

Previous studies have shown that various countermeasures, including dense compaction, prewetting, and chemical stabilization, can largely eliminate loess collapsibility to meet the requirements of strength and deformation as a foundation $[2,3]$. However, the engineering properties of treated loess soils are easily influenced by the long-term strong weathering processes such as freeze-thaw (FT) and/or wetting-drying (WD) cycles [4]. Hence, these weathering processes may put infrastructures built on treated loess soils at risk of instability or failure due to excessive deformation produced.

Numerous investigations have been conducted to understand the effects of FT cycles on the physical and mechanical properties of soils. Zhang et al. pointed out that FT cycles can induce the fragmentation of soil coarse particles and the aggregation of fine particles leading to the change in soil structure [5]. Chamberlain and Gow showed that the hydraulic conductivity of soils increases by one or two orders of magnitude after FT cycles [6]. The FT cycles have a dual effect on the void ratio of soils as follows: loose soils become denser while dense soils become looser, and both loose and dense soils will have the same void ratio after 
several FT cycles $[7,8]$. The mechanical properties of soils show different trends under freezing-thawing weathering because of different soil types, densities, and methods of the FT cycle test [9]. Mu et al. indicated that the microstructure of soils changes significantly under cyclic freeze-thaw conditions, the contents of macro- and midpores can increase, and the bonding of soil particles can partially change from face-to-face contact to point-to-point contact after a few FT cycles [10].

The wetting-drying process can lead to large variations in the strength, permeability, and deformation of fine-grained soils including the loess soils. All these changes are due to the modification of the size, shape, and arrangement of loess particles [11], destruction of pore structures [12, 13], change in interparticle bonding forces [13], decrystallization and crystallization of soluble salts [14], and shrinking and swelling of clay minerals. Generally, the static and dynamic strength of soils decreases obviously in the first few WD cycles and remains almost unchanged with additional WD cycles [15-17]. The cumulative deformation and permeability coefficient of soils can increase with an increasing number of WD cycles $[18,19]$. The release and transport of loess colloidal particles can also increase with the increasing number of the WD cycle [20, 21].

In the Loess Plateau, loess foundations or subgrades are often subjected to combined FT and WD weathering. Little attention, however, has been paid to the effects of repeated wetting-drying-freezing-thawing (WDFT) cycles on the geotechnical properties of soils [22]. In the present paper, a series of laboratory experiments, including oedometer tests, direct shear tests, and mercury intrusion porosimetry (MIP) tests, have been carried out to investigate the effects of WDFT cycles on the deformation behaviors and pore characteristics of compacted loess.

\section{Materials and Methods}

2.1. Materials. The loess soil used in this investigation was collected in Yongdeng County $\left(36^{\circ} 36^{\prime} 34^{\prime \prime} \mathrm{N}\right.$ and $103^{\circ} 22^{\prime} 05^{\prime \prime}$ E), Gansu Province at western Loess Plateau of China. This loess was characterized by high collapse potential [14]. Figure 1 presents the particle size distribution of the loess. It can be observed that the loess consists predominantly of clay and silt particles, accounting for $66.1 \%$ and $19.2 \%$, respectively. The particle size distribution curve shows two peaks at particle sizes of about 0.08 and $35 \mu \mathrm{m}$, respectively. Some basic physical properties of the loess soils are given in Table 1. The main chemical compositions of the collapsible loess are $\mathrm{SiO}_{2}, \mathrm{CaCO}_{3}$, and $\mathrm{Al}_{2} \mathrm{O}_{3}$, accounting for $51.5 \%$, $12.7 \%$, and $10.5 \%$, respectively (Table 2 ).

2.2. Preparation of Compacted Loess Specimen. The sampled loess soils were air-dried naturally and crushed to pass through a $2 \mathrm{~mm}$ sieve to ensure good mixing. To achieve the optimum water content of 13\% (Table 1), the required amount of deionized water was added to the air-dried loess using a spray bottle. Then, the wetted loess was kept in a sealed plastic bag for $24 \mathrm{~h}$ to reach moisture equilibrium.

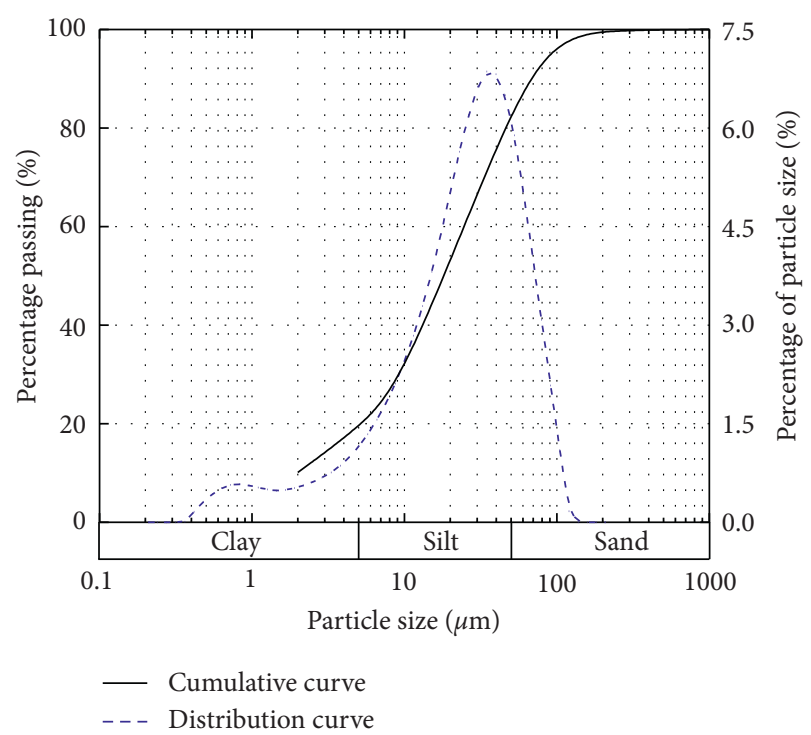

FIgURE 1: Grain size distribution curve of the loess.

Table 1: Physical properties of the loess.

\begin{tabular}{lc}
\hline Parameter & Value \\
\hline Specific gravity & 2.70 \\
Liquid limit (\%) & 26.29 \\
Plastic limit (\%) & 18.24 \\
Plasticity index (\%) & 8.05 \\
Optimum moisture content $(\%)$ & 13.0 \\
Maximum dry density $\left(\mathrm{g} / \mathrm{cm}^{3}\right)$ & 1.912 \\
Total soluble salt content $(\%)$ & 0.8 \\
\hline
\end{tabular}

TAble 2: Main chemical components of the loess.

\begin{tabular}{lc}
\hline Chemistry & Content (\%) \\
\hline Silicon oxide $\left(\mathrm{SiO}_{2}\right)$ & 51.5 \\
Calcium carbonate $\left(\mathrm{CaCO}_{3}\right)$ & 12.7 \\
Aluminum oxide $\left(\mathrm{Al}_{2} \mathrm{O}_{3}\right)$ & 10.5 \\
Calcium oxide $(\mathrm{CaO})$ & 7.1 \\
Ferric oxide $\left(\mathrm{Fe}_{2} \mathrm{O}_{3}\right)$ & 2.8 \\
Magnesium oxide $(\mathrm{MgO})$ & 2.1 \\
Potassium oxide $\left(\mathrm{K}_{2} \mathrm{O}\right)$ & 1.8 \\
Ferrous oxide $(\mathrm{FeO})$ & 1.8 \\
\hline
\end{tabular}

Finally, the loess was put in a cylindrical steel mold and densely compacted to a density of $1.81 \mathrm{~g} / \mathrm{cm}^{3}$, producing specimens with a diameter of $61.8 \mathrm{~mm}$ and a height of $20 \mathrm{~mm}$.

2.3. Wetting-Drying-Freezing-Thawing Cycle. The Yongdeng County has a temperate continental climate with a monthly average air temperature ranging from $-14^{\circ} \mathrm{C}$ in January to $26^{\circ} \mathrm{C}$ in July. We measured the volumetric water content of compacted loess in the shallow layer of the embankment near the sampling site and found that it ranged from $5 \%$ to $29 \%$. Therefore, the backfilled loess was subjected to combined FT and WD cycles. In this study, the specimens 
underwent WDFT processes as follows: the prepared specimens in Section 2.2 were first air-dried to $1 \%$ water content at room temperature. Next, the specimens were wetted to the saturated water content of $18 \%$ by dripping water using a burette. After that, the specimens were wrapped by plastic membranes and placed in sealed polyethylene bags to prevent water loss throughout the FT cycles, frozen at $-15^{\circ} \mathrm{C}$ for $12 \mathrm{~h}$, and then thawed at $16^{\circ} \mathrm{C}$ for another $12 \mathrm{~h}$. Finally, the specimens were dried naturally to the target water content of $13 \%$. Each WDFT cycle contained a WD cycle and an FT cycle. The specimens were subjected to 1, 3, 5 , or 7 WDFT cycles.

2.4. Experimental Methods. When one or several WDFT cycles were reached, compression tests, collapsibility tests, direct shear tests, and MIP tests were performed on the loess specimens according to testing methods of soils for highway engineering [23]. For the sake of comparison, the same geotechnical tests were also carried out on specimens not subjected to any weathering.

Compression tests were conducted on compacted loess specimens exposed to a given weathering intensity with the optimum water content of $13 \%$ at loads of $50,100,150,200$, $300,400,800$, and $1600 \mathrm{kPa}$, using a GZQ-1 oedometer apparatus. When the measured vertical settlement for a given load was less than $0.01 \mathrm{~mm}$ per hour, it was assumed that the compression deformation was stabilized at this load, and then, a new load was applied. Double-oedometer tests were used in this study for investigating the collapsibility of specimens. Two nominally identical specimens were tested under four loads, i.e., $50,100,200$, and $300 \mathrm{kPa}$, in the GZQ1 oedometer. One with a water content of $13 \%$ was tested to give an unsaturated compression curve. The other one was inundated with water at a load of $50 \mathrm{kPa}$ when the measured compression deformation was less than $0.01 \mathrm{~mm}$ per hour, and then, the load was increased up to $300 \mathrm{kPa}$, for testing the saturated compression curve. It should be noted that, during the above tests, the unsaturated loess specimens should be covered with cotton gauze to prevent excessive evaporation which could lead to erroneous results [23]. Quick shear tests were carried out with four compacted loess specimens with a constant water content of $13 \%$ using normal pressures of $50,100,150$, and $200 \mathrm{kPa}$ after each WDFT cycle in the strain-controlled direct shear apparatus. The shear strain-controlled rate was $0.8 \mathrm{~mm} / \mathrm{min}$ in these tests. The tests ceased when the shear displacement reached $6 \mathrm{~mm}$

MIP tests were performed on the cube specimens using an AutoPore IV 9520 porosimeter to measure the quantitative distribution of pores within the compacted loess under different WDFT cycles. The cube specimens, having the dimensions of approximately $1 \mathrm{~cm}^{3}$, were trimmed out from the central part of the aforementioned air-dried cylindrical specimens.

\section{Experimental Results}

3.1. Compression Deformation. The secant modulus method based on the vertical compression strain, proposed by Wei [24], was used to directly describe the compression behavior of compacted loess soils. The vertical compression strain $\varepsilon_{i}$ can be calculated by the following equation, where $h_{0}$ is the initial height of the specimen and $h_{i}$ is the stabilized height at a given load:

$$
\varepsilon_{i}=\frac{h_{0}-h_{i}}{h_{0}} \times 100 \%
$$

Figure 2 shows the vertical compression strain of compacted loess specimens with the same water content of $13 \%$ as a function of load under different WDFT weathering. The vertical compression strain increases rapidly with the increasing load when the applied load is below $400 \mathrm{kPa}$ and then increases slowly for loads from 400 to $1600 \mathrm{kPa}$ due to the densification of loess. As the number of WDFT cycles increases, the vertical compression strains of loess specimens are all increased at different loads, indicating that the WDFT cycles have a strong effect on the compression behavior of loess soil.

Based on the analysis of the numerous experimental data, Liu et al. pointed out that the relationship between vertical compression strain $\left(\varepsilon_{i}\right)$ and load $\left(p_{i}\right)$ for common soils can be described with hyperbolic function [25], written as equation (2). The compression test data are fitted using equation (2), and the fitting parameters $\mathrm{A}$ and $\mathrm{B}$ are summarized in Table 3. From the results summarized, it can be seen that the hyperbola model can describe the relationship between $\varepsilon_{i}$ and $p_{i}$ under different WDFT cycles, reasonably, with $R^{2}$ higher than 0.994 . Parameters $\mathrm{A}$ and $\mathrm{B}$ are significantly reduced before and after the WDFT treatment, and they gradually decrease with an increasing number of WDFT cycles. For instance, the value of the constant B decreases from 17.47 for intact loess specimens to 11.60 after the first cycle and to 6.79 after 7 WDFT cycles:

$$
\varepsilon_{i}=\frac{p_{i}}{A+B p_{i}} .
$$

Secant modulus is defined herein as the ratio of the load and the vertical compression strain, i.e., $E_{o_{i}}=p_{i} / \varepsilon_{i}$. The variations in secant modulus of compacted loess specimens at loads of $100,200,300$, and $400 \mathrm{kPa}$ as a function of the number of WDFT cycles are shown in Figure 3. The secant modulus decreases rapidly during the first 3 WDFT cycles and then remains constant at all loads. Besides, the relationship between the secant modulus and the number of WDFT cycles $n_{i}$ can be well fitted by a power function (i.e., equation (3)) with $R^{2}$ higher than 0.969; the fitting parameters $\alpha$ and $\beta$ increase linearly with the load increasing, and $\gamma$ changes negligibly:

$$
E_{o_{i}}=\alpha+\beta e^{\gamma n_{i}} .
$$




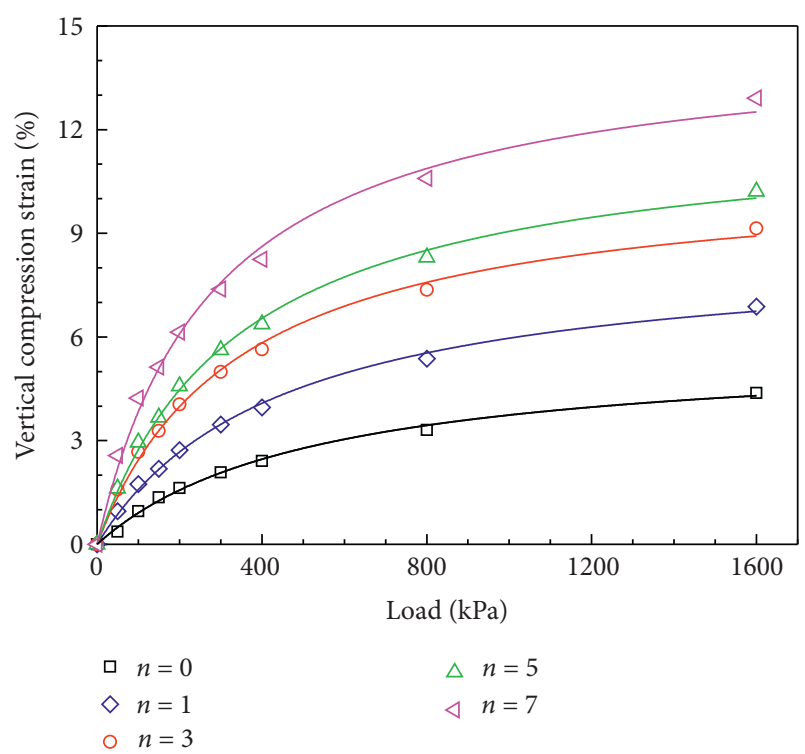

Figure 2: Curves of vertical compression strain and load at different wetting-drying-freezing-thawing cycles.

TABLE 3: Fitting parameters of equation (2) for the data of compression tests.

\begin{tabular}{lccc}
\hline Number of cycles & A & B & $R^{2}$ \\
\hline 0 & 9257.26 & 17.47 & 0.994 \\
1 & 5164.09 & 11.60 & 0.997 \\
3 & 3173.47 & 9.23 & 0.996 \\
5 & 2833.08 & 8.21 & 0.998 \\
7 & 1927.11 & 6.79 & 0.994 \\
\hline
\end{tabular}

3.2. Collapse Deformation. In the Chinese code for building construction in collapsible loess regions [26], the coefficient of collapsibility $\delta_{s}$ is employed to quantify the degree of collapsibility of loess soils and is defined as equation (4), where $h_{0}$ denotes the initial height of the loess specimens and $h_{p}$ and $h_{p}^{\prime}$ represent the stabilized height of unsaturated and saturated soil specimens at a given load, respectively. As a rule, the loess soil is recognized as a collapsible soil for $\delta_{s}>0.015$ at the load of $200 \mathrm{kPa}$ :

$$
\delta_{s}=\frac{h_{p}-h_{p}^{\prime}}{h_{0}}
$$

Figure 4 shows the relations between $\delta_{s}$ of compacted loess specimens and the vertical pressure for different intensities of WDFT weathering obtained by the doubleoedometer tests. It can be seen that the coefficient of collapsibility gradually increases with an increasing number of WDFT cycles at all loads. For instance, the coefficients of collapsibility increase from an initial value of 0 for intact loess specimens to 0.013 after 5 WDFT cycles. Interestingly, after 7 WDFT cycles, the coefficient of collapsibility exceeds the critical value of 0.015 at a load of $200 \mathrm{kPa}$, indicating that

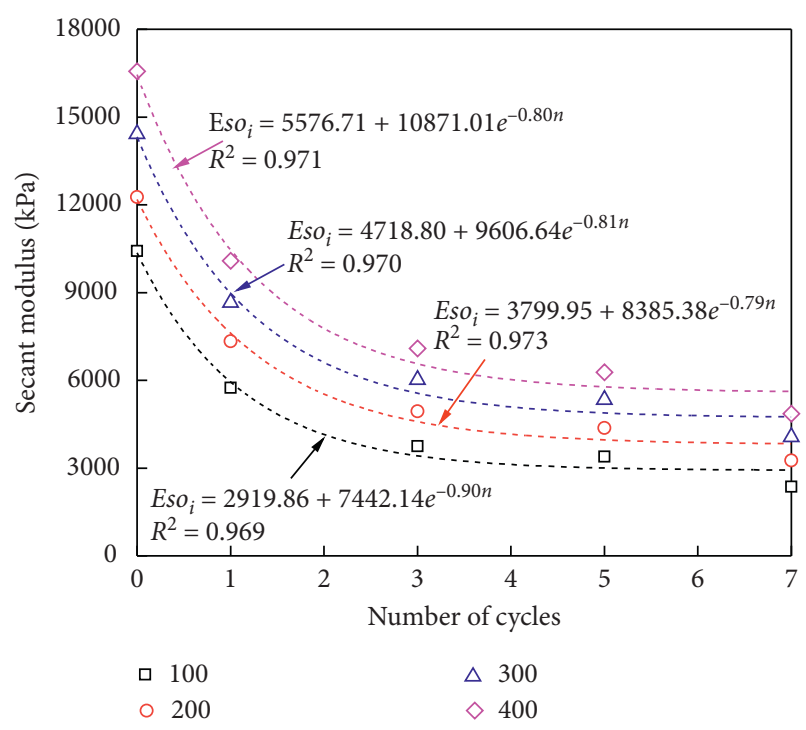

FIGURE 3: Relationships between secant modulus and the number of wetting-drying-freezing-thawing cycles.

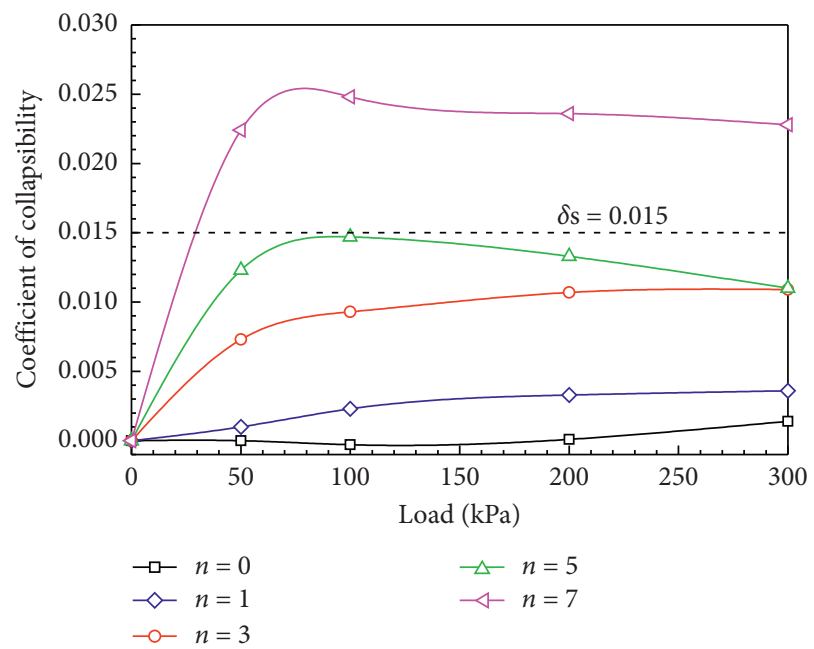

FIGURE 4: Coefficient of collapsibility of compacted loess specimens as a function of load at different wetting-drying-freezing-thawing cycles.

the compacted loess specimens exhibit secondary collapse. This is mainly attributed to a net increase in volume, a loose structure, and a decrease in the salt content [14].

3.3. Shear Strength. Table 4 presents the shear strength of loess specimens at loads of $50,100,150$, and $200 \mathrm{kPa}$ obtained from the direct shear tests, as well as the cohesion and internal friction angle, at different WDFT cycles. The cohesion gradually decreases with an increase in the number of WDFT cycles. For instance, the cohesion decreases by $37.9 \%$ 
TABLE 4: Results of the direct shear test for compacted loess specimens.

\begin{tabular}{|c|c|c|c|c|c|c|}
\hline \multirow{2}{*}{ Number of cycles } & \multicolumn{4}{|c|}{ Shear strength $(\mathrm{kPa})$} & \multirow{2}{*}{ Cohesion $(\mathrm{kPa})$} & \multirow{2}{*}{ Internal friction angle $\left({ }^{\circ}\right)$} \\
\hline & $50 \mathrm{kPa}$ & $100 \mathrm{kPa}$ & $150 \mathrm{kPa}$ & $200 \mathrm{kPa}$ & & \\
\hline 0 & 83.05 & 123.05 & 155.30 & 200.50 & 44.3 & 37.6 \\
\hline 1 & 72.89 & 116.43 & 158.65 & 187.31 & 37.5 & 36.3 \\
\hline 3 & 68.62 & 95.60 & 130.62 & 177.77 & 27.5 & 36.0 \\
\hline 5 & 68.71 & 92.85 & 148.44 & 172.86 & 29.2 & 37.7 \\
\hline
\end{tabular}

from an initial value of $44.3 \mathrm{kPa}$ to $27.5 \mathrm{kPa}$ after $3 \mathrm{WDFT}$ cycles and then remains nearly unchanged. However, the internal friction angle has no obvious change before and after any intensity of WDFT weathering, ranged from $36.0^{\circ}$ to $37.7^{\circ}$.

3.4. Pore Distribution. In MIP tests, Washburn's equation (i.e., equation (5)), which is derived for the capillary flow of a liquid in a cylindrical tube [27], was used to determine the pore diameter $(r)$ based on the applied pressure $(P)$ needed to force the mercury into a pore against liquid surface tension, where $\sigma$ is the surface tension of mercury at $20^{\circ} \mathrm{C}$ and $\theta$ is the solid-liquid contact angle. In this paper, a solidliquid contact angle of $130^{\circ}$ and a surface tension of $0.485 \mathrm{~N} / \mathrm{m}$ were used in the calculations:

$$
r=-\frac{4 \sigma \cos \theta}{P}
$$

Figure 5 presents the pore size distribution in terms of cumulative pore volume and pore size density of compacted loess specimens exposed to different intensities of WDFT cycles. The distribution is shown for a diameter range of 0.003 to $1000 \mu \mathrm{m}$. Figure 5(a) depicts that all cumulative intrusion curves are essentially flat at diameters less than $0.01 \mu \mathrm{m}$, indicating all of the pores in compacted loess have a diameter larger than $0.003 \mu \mathrm{m}$. Theoretically, the measured total mercury intrusion volume of loess specimens should be consistent with the void ratio. It, therefore, increases with an increase in the number of WDFT cycles. For instance, the total mercury intrusion volume increases from an initial value of $0.17 \mathrm{~mL}^{-1} \mathrm{~g}^{-1}$ for intact loess specimens to $0.25 \mathrm{~mL} \cdot \mathrm{g}^{-1}$ after 3 WDFT cycles. However, the abnormal variation in total mercury intrusion volume occurs after 5 WDFT cycles. This is due to the randomness of specimens selected for the MIP test [28]. The pore size density functions show the pore size in compacted loess is chiefly concentrated in $0.01 \sim 100 \mu \mathrm{m}$ diameter, and the pores with a diameter of $1 \sim 35 \mu \mathrm{m}$ are significantly influenced by the WDFT cycles (Figure 5(b)).

According to the measured distribution of pore size and grain size of the compacted loess (Figure 1) and the classification of loess pores proposed by Lei [29], we herein classify loess pores into four types based on the equivalent pore diameter: the large pores $(>35 \mu \mathrm{m})$, the medium pores $(35 \sim 10 \mu \mathrm{m})$, the small pores $(10 \sim 0.8 \mu \mathrm{m})$, and the micropores $(<0.8 \mu \mathrm{m})$, to provide a quantitative analysis of the effects of WDFT cycles on the various pores. Pore contents of the compacted loess specimens at different WDFT cycles are calculated and given in Figure 6. Note that the WDFT weathering decreases the volumes of small and micropores but significantly increases the medium pore volume, without changing the large pore volume, eventually leading to an increase in mean pore diameter. Particularly, the medium pore content increases from $1.04 \%$ for the intact specimens to $11.2 \%$ after 3 WDFT cycles. The results suggest that, with the increase in the number of WDFT cycles, the small and micropores may convert into medium pores. Moreover, the medium pore has a major influence on the secondary collapse of compacted loess [30]. Therefore, the compacted loess specimens exhibit secondary collapse in this study (Figure 4).

\section{Discussion}

The aforementioned results demonstrate that the WDFT weathering has a stronger deterioration effect on the geotechnical properties of densely compacted loess, which is manifested by increased deformation, decreased strength, and an obvious change in soil microstructure. This is consistent with the mesostructure evolution of compacted loess exposed to WDFT cycles reported by Chen [31]. All indicate that the variation trends of macromechanical parameters and micro- and mesoscopic structures in compacted loess are consistent.

To help in comparison with the deterioration effects of $\mathrm{WD}, \mathrm{FT}$, and WDFT weathering, the variations in vertical compression strain and coefficient of collapsibility of compacted loess specimens at a load of $200 \mathrm{kPa}$ as a function of weathering intensity are shown in Figure 7. The vertical compression strain increases considerably with the increasing number of WD and WDFT cycles while it is not affected at all by the FT cycles. These results suggest that the WD and WDFT weathering have a much stronger effect on the compression behavior of loess soils than FT weathering. Besides, in the first 3 cycles, the vertical compression strains of loess specimens subjected to WD and WDFT weathering are equal. However, these deformations are much larger for loess specimens exposed to WD weathering than those exposed to WDFT weathering after 5 cycles (Figure 7(a)). As illustrated in Figure 7(b), the coefficient of collapsibility shows the same changing rule as the vertical compression strain under WD, FT, and WDFT cycling. It also gradually increases following the WD and WDFT cycles while it is hardly affected by the FT cycles. After 5 WD and 7 WDFT cycles, the compacted loess specimens exhibit secondary collapse probably due to a significant increase in the medium pore content (Figure 6).

As mentioned above, it can be concluded that dry-wet action plays a dominant role in increasing loess deformation 


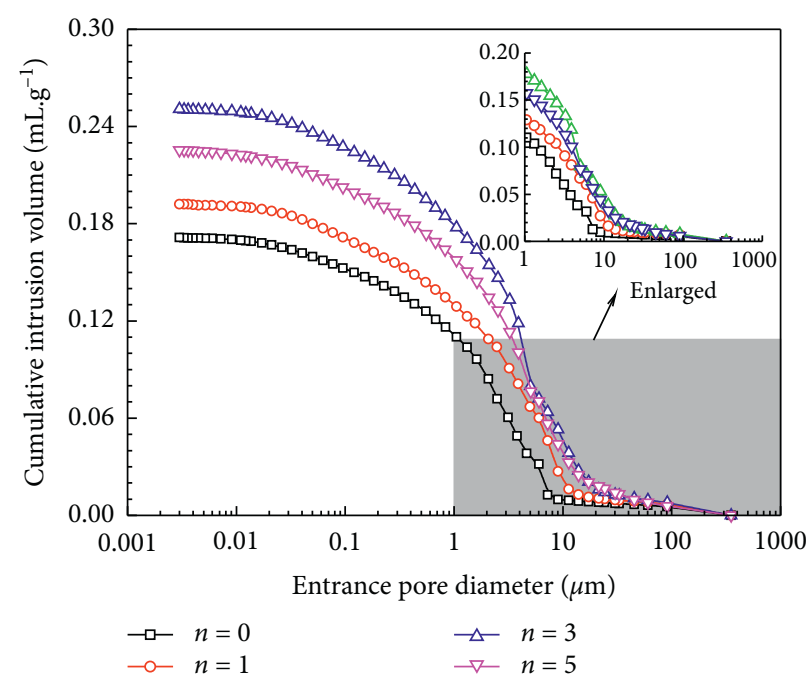

(a)

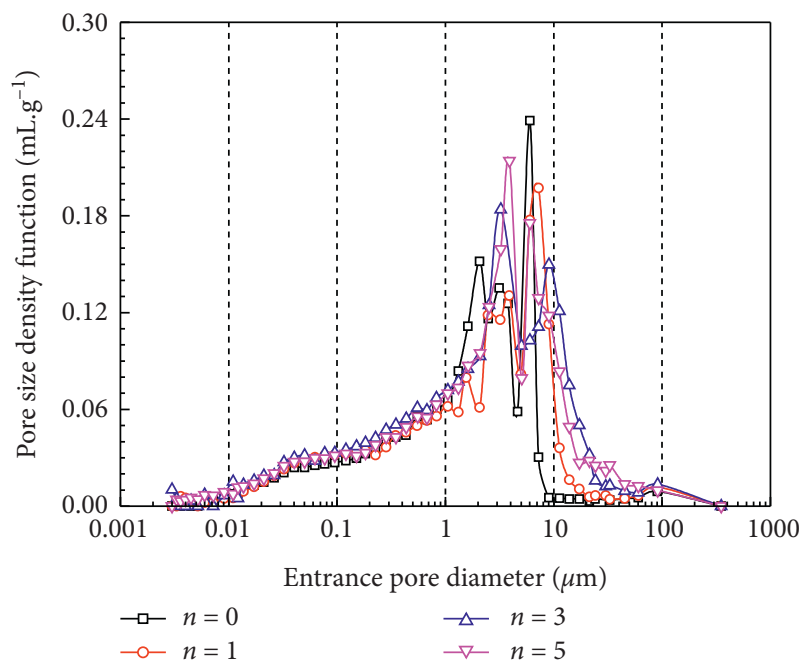

(b)

FiguRE 5: MIP test results on compacted loess specimens subjected to different numbers of wetting-drying-freezing-thawing cycles. (a) Cumulative intrusion curve. (b) Pore size density functions.
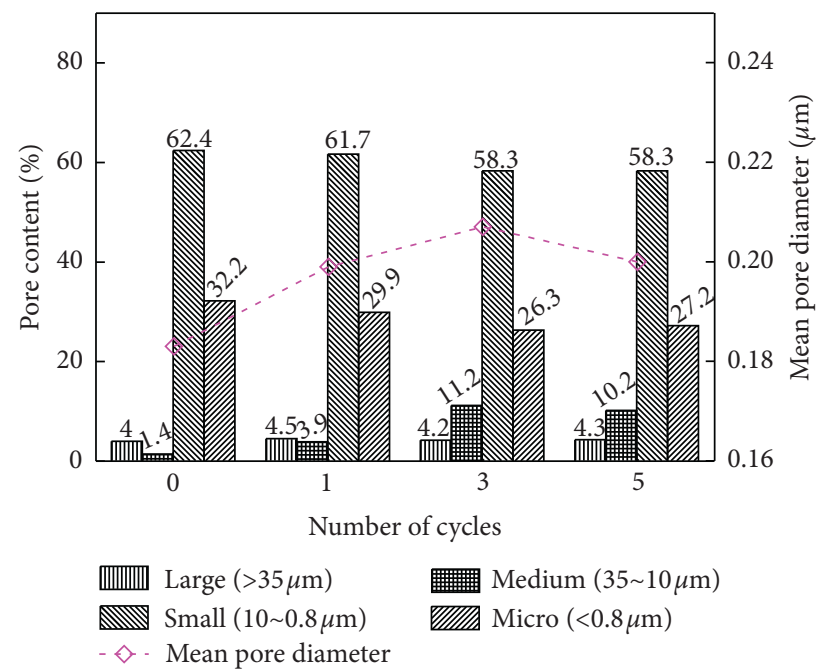

FIGURE 6: Variation in pore content and mean pore diameter of compacted loess specimens subjected to wetting-drying-freezing-thawing cycles.

during the WDFT cycles, mainly due to the high sensitivity of loess to water than the temperature [14]. However, freezethaw action restraints the deformation growth to some extent after 5 WDFT cycles in this study. This phenomenon can be explained by the residual void ratio and a corresponding residual dry density for soils subjected to FT cycles proposed by Viklander [7] and further confirmed by Li et al. [8]. In the first 3 cycles, the dry density of compacted loess specimens gradually decreases with an increasing number of
WDFT weathering. With the increase in the number of WDFT cycles, freeze-thaw action begins to inhibit this decreasing trend when the density further reduces to residual dry density.

Although the collapsible loess can meet the requirements of strength and deformation by dense compaction, large deformation and secondary collapse still occur in compacted loess specimens during WDFT weathering. Therefore, the influences of WDFT weathering should be taken into 


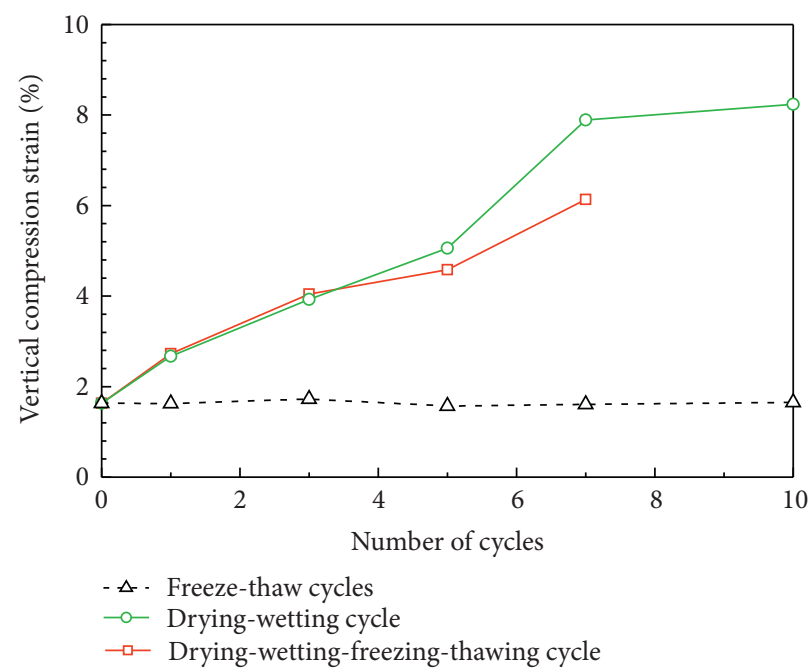

(a)

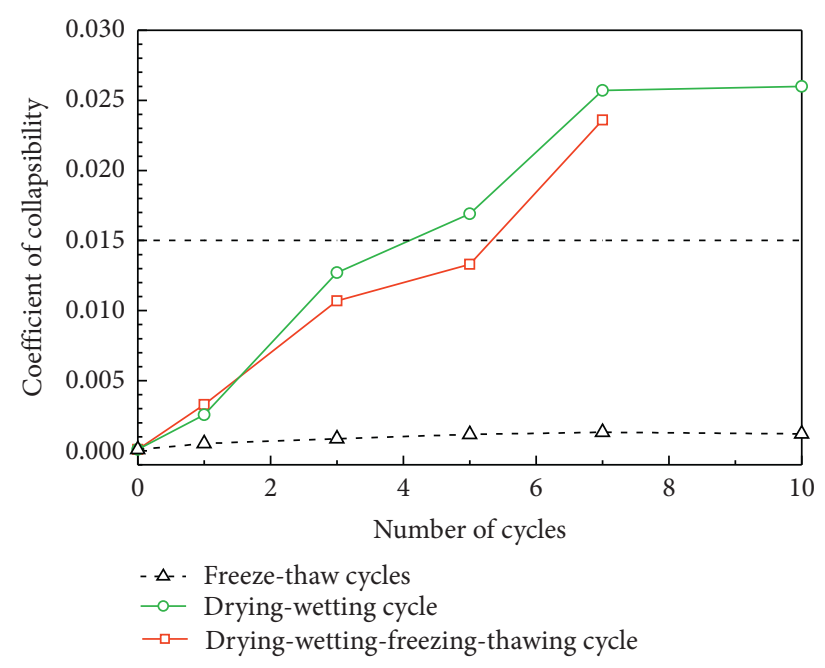

(b)

FIGURE 7: Variation in vertical compression strain (a) and coefficient of collapsibility (b) of compacted loess specimens at a load of $200 \mathrm{kPa}$ during wetting-drying, freeze-thaw, and wetting-drying-freezing-thawing cycles.

account for the prediction of the long-term stability of infrastructures built on loess soils in the arid and seasonally frozen ground regions.

\section{Conclusions}

In this paper, the deterioration effect of WDFT weathering on the mechanic properties of compacted loess was assessed based on the results of oedometer tests, direct shear tests, and MIP tests, and the following conclusions can be drawn:

(1) The WDFT cycles have a significant influence on the mechanic behavior of compacted loess. The vertical compression strain and coefficient of collapsibility of compacted loess specimens gradually increase with an increasing number of WDFT cycles while the secant modulus and cohesion decrease. After 7 WDFT cycles, the compacted loess specimens begin to exhibit secondary collapse.

(2) The compacted loess specimens consist predominantly of pores smaller than $10 \mu \mathrm{m}$, and the pores with a diameter of $1 \sim 35 \mu \mathrm{m}$ are greatly influenced by the WDFT cycles. As the number of WDFT cycles increases, small and micropores may convert into medium pores, and thus, the medium pore content increases more than tenfold.

(3) A comparison among the deformation properties of compacted loess specimens after WD, FT, and WDFT cycles shows that dry-wet action plays a dominant role in increasing loess deformation during the WDFT cycles, and freeze-thaw action restraints the deformation growth after 5 WDFT cycles.

\section{Data Availability}

The data used to support the finding of this study are available from the corresponding author upon request.

\section{Conflicts of Interest}

The authors declare that they have no conflicts of interest.

\section{Acknowledgments}

This work was supported by the Strategic Priority Research Program of Chinese Academy of Sciences (no. XDA2003020102), the National Natural Science Foundation of China (nos. 41672310 and 41630636), and the Science and Technology Major Project of Gansu Province (no. 143GKDA007).

\section{References}

[1] G. Y. Li, W. Ma, F. Wang et al., "Processes and mechanisms of multi-collapse of loess roads in seasonally frozen ground regions: a review," Sciences in Cold and Arid Regions, vol. 7, pp. 456-468, 2015.

[2] K. M. Rollins and G. W. Rogers, "Mitigation measures for small structures on collapsible alluvial soils," Journal of Geotechnical Engineering, vol. 120, no. 9, pp. 1533-1553, 1994.

[3] C. L. Chen, P. GAO, and Z. Q. Hu, "Moistening deformation characteristic of loess and its relation to structure," Chinese Journal of Rock Mechanics and Engineering, vol. 25, pp. 1352-1360, 2006.

[4] S.-L. Wang, Q.-F. Lv, H. Baaj, X.-Y. Li, and Y.-X. Zhao, "Volume change behaviour and microstructure of stabilized loess under cyclic freeze-thaw conditions," Canadian Journal of Civil Engineering, vol. 43, no. 10, pp. 865-874, 2016. 
[5] Z. Zhang, W. Ma, W. Feng, D. Xiao, and X. Hou, "Reconstruction of soil particle composition during freeze-thaw cycling: a review," Pedosphere, vol. 26, no. 2, pp. 167-179, 2016.

[6] E. J. Chamberlain and A. J. Gow, "Effect of freezing and thawing on the permeability and structure of soils," Developments in Geotechnical Engineering, vol. 13, pp. 73-92, 1979.

[7] P. Viklander, "Permeability and volume changes in till due to cyclic freeze/thaw," Canadian Geotechnical Journal, vol. 35, no. 3, pp. 471-477, 1998.

[8] G.-Y. Li, W. Ma, Y.-H. Mu, F. Wang, S.-Z. Fan, and Y.-H. Wu, "Effects of freeze-thaw cycle on engineering properties of loess used as road fills in seasonally frozen ground regions, North China," Journal of Mountain Science, vol. 14, no. 2, pp. 356-368, 2017.

[9] J. Qi, P. A. Vermeer, and G. Cheng, "A review of the influence of freeze-thaw cycles on soil geotechnical properties," Permafrost and Periglacial Processes, vol. 17, no. 3, pp. 245-252, 2006.

[10] Y. H. Mu, W. Ma, G. Y. Li, and Y. C. Mao, "Quantitative analysis of impacts of freeze-thaw cycles upon microstructure of compacted loess," Chinese Journal of Geotechnical Engineering, vol. 33, pp. 1919-1925, 2011.

[11] W. K. Ni, K. Z. Yuan, X. F. Lü, and Z. H. Yuan, "Comparison and quantitative analysis of microstructure parameters between original loess and remolded loess under different wetting-drying cycles," Scientific Reports, vol. 10, no. 1, p. $5547,2020$.

[12] H. J. Lu, J. X. Li, W. W. Wang, and C. H. Wang, "Cracking and water seepage of Xiashu loess used as landfill cover under wetting-drying cycles," Environmental Earth Sciences, vol. 74, pp. 1-10, 2015.

[13] L. F. Pires, A. C. Auler, W. L. Roque, and S. J. Mooney, "X-ray microtomography analysis of soil pore structure dynamics under wetting and drying cycles," Geoderma, vol. 362, Article ID 114103, 2020.

[14] G. Li, F. Wang, W. Ma et al., "Variations in strength and deformation of compacted loess exposed to wetting-drying and freeze-thaw cycles," Cold Regions Science and Technology, vol. 151, pp. 159-167, 2018.

[15] B. D. Kay and A. Dexter, "The influence of dispersible clay and wetting/drying cycles on the tensile strength of a red-brown earth," Soil Research, vol. 30, no. 3, pp. 297-310, 1992.

[16] H. T. Liu, A. J. Zhang, T. Duan, J. B. Lian, and X. H. Dong, "The influence of alternate dry-wet on the strength and permeability of remolded loess," Hydro-Science and Engineering, vol. 4, pp. 38-42, 2010.

[17] T. X. Wang, Y. Z. Hao, Z. Wang, L. Cheng, and J. L. Li, "Experimental study on dynamic strength properties of compacted loess under wetting-drying cycles," Chinese Journal of Rock Mechanics and Engineering, vol. 39, pp. 1242-1251, 2020.

[18] M. A. Malusis, S. Yeom, and J. C. Evans, "Hydraulic conductivity of model soil-bentonite backfills subjected to wetdry cycling," Canadian Geotechnical Journal, vol. 48, no. 8, pp. 1198-1211, 2011.

[19] F. Wang, G. Y. Li, Y. H. Mu, P. Zhang, Y. H. Wu, and S. Z. Fan, "Experimental study of deformation characteristics of compacted loess subjected to wetting-drying cycle," Rock and Soil Mechanics, vol. 37, pp. 2306-2312, 2016.

[20] B. Zhou, Y. Wu, J. Chan, S. Wang, Z. Qiao, and S. Hu, "Wetting-drying cycles enhance the release and transport of autochthonous colloidal particles in Chinese loess," Human and Ecological Risk Assessment: An International Journal, vol. 25, no. 1-2, pp. 335-353, 2019.

[21] B. Zhou, Y. G. Wu, Z. H. Zhang, S. C. Wang, J. W. Chan, and S. H. Hu, "Laboratory tests on effects of wetting-drying cycles and loess layer thickness on release and transport of loess colloidal particles in artificial loess columns," Environmental Earth Sciences, vol. 78, no. 15, p. 478, 2019.

[22] Z. X. Zeng and L. W. Kong, "Effect of wetting-dryingfreezing-thawing cycles on the swelling behaviour of the Yanji mudstone," Environmental Earth Sciences, vol. 78, p. 435, 2019.

[23] Ministry of Transport of the People's Republic of China, Test Methods of Soils for Highway Engineering, JTG E40-2007, China Communication Press, Beijing, China, 2007.

[24] R. L. Wei, "A new method to sort the data of the compression test," Journal of Nanjing Hydraulic Research Institute, vol. 12, no. 1, pp. 90-93, 1980.

[25] B. J. Liu and J. L. Zhang, "Application and analyzing method for soil compression test," China Journal of Highway and Transport, vol. 12, pp. 37-41, 1999.

[26] Ministry of Construction of the People's Republic of China, Code for Building Construction in Collapsible Loess Regions, GB 50025-2004, Architecture and Building Press, Beijing, China, 2004.

[27] E. W. Washburn, "Note on a method of determining the distribution of pore sizes in a porous material," Proceedings of the National Academy of Sciences, vol. 7, no. 4, pp. 115-116, 1921.

[28] K. B. Ren, B. Wang, X. M. Li, and S. Yin, "Influence of the compaction procedure on mechanical behaviors and pore characteristics of silts," Chinese Journal of Rock Mechanics and Engineering, vol. 38, pp. 842-851, 2019.

[29] X. Y. Lei, "Pore types of loess soils in China and its collapsibility," Chinese Science (B section), vol. 12, p. 1310e6, 1987.

[30] P. Li, S. K. Vanapalli, and T. L. Li, "Review of collapse triggering mechanism of collapsible soils due to wetting," Journal of Rock Mechanics and Geotechnical Engineering, vol. 8, pp. 1-18, 2016.

[31] S. J. Chen, W. Ma, and G. Y. Li, "Study on the mesostructural evolution mechanism of compacted loess subjected to various weathering actions," Cold Regions Science and Technology, vol. 167, pp. 1-12, 2019. 